\title{
VIII. Kraftfahrsachverständige und Fahrlehrer - zwischen Unabhängigkeit und Vereinnahmung
}

Im Sommer 1938 bilanzierte der Korpsführer des NSKK Adolf Hühnlein das bisher in der Motorisierungspolitik Erreichte und äußerte sich in diesem Zusammenhang unzufrieden über den Zustand des Fahrlehrergewerbes und des technischen Sachverständigenwesens. Um „Missstände“ in diesen Bereichen zu beseitigen, sei es notwendig, so Hühnlein, die Fahrlehrer unter die Aufsicht des Korps zu stellen und die Kraftfahrsachverständigen aus den „überlebten Vereinen“ herauszulösen. Als oberstes Gebot nannte er für die genannten Berufsgruppen „politische Zuverlässigkeit" und suggerierte damit Loyalitätsdefizite bei den Trägern der Fahrausbildung und den Sachverständigen gegenüber dem nationalsozialistischen Staat. ${ }^{1}$ Die Neuordnungspläne des NSKK wurden jedoch nur teilweise verwirklicht. Anhand der Auseinandersetzungen, die das NSKK mit den Technischen Überwachungs- und Fahrlehrer-Vereinen und sie betreffend auch mit anderen Institutionen in Staat und Partei führte, werden die Möglichkeiten und Grenzen der Machtausweitung des NSKK deutlich.

\section{Tradition und Neuordnung im Technischen Prüfwesen}

Mit der Gründung des Zentral-Verbandes der Preußischen Dampfkessel-Überwachungs-Vereine im Jahre 1886 übernahm die Wirtschaft die Verantwortung für den gefahrenfreien Betrieb ihrer technischen Anlagen. Namhafte Industrievertreter gründeten Dampfkessel-Überwachungs- oder Revisionsvereine (später: Technische Überwachungsvereine), deren Kennzeichen die privatrechtliche Organisation in Vereinen war. Ein immer größer werdendes Aufgabengebiet bildeten die Überprüfung von Kraftfahrzeugen, die Abnahme von Führerscheinprüfungen und die Mitwirkung bei der Zulassung von Fahrlehrern. Die Überwachungskompetenz der Sachverständigen trat im „Dritten Reich“ fast zwangsläufig in Konflikt mit dem staatlichen Anspruch auf Regulierungskompetenz. Da die Technischen Überwachungsvereine seit jeher vor „staatlicher Reglementierungssucht“ ${ }^{2}$ warnten und den Grundsatz der Selbstverwaltung der Wirtschaft verteidigten, war eine Kollision mit den nationalsozialistischen Ordnungsprinzipien vorhersehbar.

Anfänglich zeigte sich bei den Repräsentanten der Technischen Überwachungsvereine, deren Mitarbeiter alle eine Ingenieurausbildung absolviert hatten, eine große Genugtuung über die politische und gesellschaftliche Akzeptanz, die der

1 Vgl. Krafthand vom 6. 8. 1938: „Es ist ein großer Stolz, NSKK-Mann zu sein!“

2 Hoffmann: Unabhängig und neutral, Vorwort. 
Technik im nationalsozialistischen Staat zugesprochen wurde. ${ }^{3}$ Die Verbandszeitschrift des Bayerischen Revisions-Vereins von 1938 begrüßte anlässlich der Gründung des Hauses der Deutschen Technik den Statusgewinn der Ingenieure:

„Noch niemals hat die Technik solche Anerkennung gefunden wie im Dritten Reiche. Sie steht nicht mehr zweitrangig hinter anderer geistiger Arbeit; sie ist ebenbürtig, mehr noch, Symbol unserer Zeit, das vom ganzen Volke verstanden und freudig bejaht wird. Waren in all den Jahrzehnten die Techniker und ihre Werke von einer materialistischen Wirtschaft mißbraucht worden, so steigerte sich die Mißachtung der schöpferischen Ingenieurleistung in den Jahren der großen Arbeitslosigkeit zu einer Diffamierung der Ingenieurschaft. Zwar sprach man vom ,Fluch der Technik', man wollte aber damit die Schaffenskraft der deutschen Techniker brechen. Ihre Arbeit drohte den Sinn zu verlieren. “4

In diesen Worten schwingt Dankbarkeit darüber mit, dass der Nationalsozialismus die Technik aus der Dienstbarmachung des Kapitalismus und aus den bürgerlichen Zwängen befreit habe. Doch auch im „Dritten Reich“ sollte die Technik der Politik dienstbar sein. Nach nationalsozialistischer Technikauffassung bestand für den Ingenieur die geistige Notwendigkeit, sich loszulösen vom „rein materialistisch-technischen Denken“, und sich bewusst zu werden, dass sich all sein Tun und Handeln „höheren, staatspolitischen Gesichtspunkten " unterzuordnen habe. ${ }^{5}$ Weiterhin musste den Ingenieuren in der NS-Zeit missfallen haben, dass die praktische Umsetzung der zur Schau gestellten Technikbegeisterung des NS-Regimes hinter den Ansprüchen zurückblieb. Es fand weder eine systematische Technikförderung statt noch ein gezielter Ausbau des Ingenieurstudiums. ${ }^{6}$

Die Ingenieure der Technischen Überwachungsvereine sahen sich spezifischen Schwierigkeiten ausgesetzt. Verschiedene Ministerien und die NSDAP nahmen bald nach der NS-Machtübernahme Anstoß an den Organisationsstrukturen der Vereine. 1934 beauftragte der Reichsverkehrsminister den Allgemeinen Verband der Deutschen Dampfkessel-Überwachungsvereine Berlin mit der Zusammenfassung der amtlich anerkannten Kfz-Sachverständigen. Die Vereine schlossen sich 1936 auf Befehl des Reichsverkehrsministers im Reichsverband der Technischen Überwachungsvereine (RTÜV) zusammen.7 1938 waren bei den Überwachungsvereinen 462 amtliche Kraftfahrsachverständige angestellt, deren Aufgabenschwerpunkte in der Abnahme von Führerscheinprüfungen und Kraftfahrzeuguntersuchungen lagen. Weitere Sonderaufträge bestanden in der Prüfung neuer Fahrzeugmodelle auf sicherheitstechnische Erfordernisse (Typprüfung), in „Fahr-

3 Dem Ingenieur wurden neben der fachlichen Führung auch politisch-ideologische Aufgaben anvertraut. Er sollte die Beziehungen zwischen Betriebsführer und Gefolgschaft harmonisieren; vgl. dazu: Walker u. a.: Technikdiskurs und Technikideologie im Nationalsozialismus, S. $440 \mathrm{ff}$.

4 Beitrag von Dr. Flemming in der Zeitschrift des Bayerischen Revisions-Vereins vom 30. 9. 1938: "Die Technik, ein wertvoller Teil des deutschen Kulturschaffens“.

5 Die Autobahn und die "Reichsautobahn“ vom 1.2. 1934: „Nationalsozialismus der Tat: Schaffung eines deutschen Volkskraftwagens" von Erwin Gottschalk.

6 Vgl. Faulenbach: Zur Einleitung in das Themenfeld „Technik und Arbeit“, S. 80.

7 Vgl. Rede des Verbandsführers auf der ersten Mitgliederversammlung des Reichsverbandes am 22. 10. 1936, in: Hoffmann: Unabhängig und neutral - die TÜV, S. 149. 
zeugmusterungen“ bei den Wehrersatzinspektionen und in der laufenden Überwachung der Lastkraftwagen für den Reichskraftwagen-Betriebs-Verband. ${ }^{8}$

In seiner Verordnung vom 19. März 1938 kündigte der Reichswirtschaftsminister gegen den Willen des RTÜV die Gliederung des Reichsgebietes in 14 Überwachungsbezirke und die Errichtung einer Reichshauptstelle für die technische Überwachung an. ${ }^{9}$ Was für die Ministerien eine Art verwaltungstechnische „Flurbereinigung" und Klärung von Zuständigkeiten bedeutete, stellte sich für den RTÜV als Generalangriff auf seine Unabhängigkeit dar. Der Verbandsführer X. Mayer kritisierte auf der Mitgliederversammlung des Reichsverbandes am 2. September 1938 die behördlichen Eingriffe in die zugestandene Selbstverwaltung und die von oben verordnete Reduzierung der ehemals 37 Vereine auf 14 Überwachungsvereine. ${ }^{10}$ Offen beklagte er die starke Einflussnahme der Ministerien im "Geist polizeilicher Bevormundung “. ${ }^{11}$ Die Leitung des RTÜV musste nicht nur dem Reichswirtschaftsministerium Folge leisten, sondern sah sich im Sommer 1938 auch mit Angriffen von Seiten der Korpsführung des NSKK konfrontiert.

\section{Der Machtkampf zwischen RTÜV und NSKK}

Der Verbandsvorsitzende des RTÜV X. Mayer erklärte sich mit einer Zusammenarbeit zwischen den Sachverständigen und dem NSKK im Bereich des Kraftfahrwesens grundsätzlich einverstanden. So bat er den Korpsführer im Dezember 1937, NSKK-Vertreter in den Beirat des RTÜV zu entsenden. NSKK-Obersturmführer Hösl trat dem technischen Ausschuss bei und Obergruppenführer Kraus saß als Vertreter des NSKK im Beirat des Reichsverbandes. ${ }^{12}$ Mayer kündigte darüber hinaus seine Bereitschaft an, unter den Sachverständigen für die Mitgliedschaft im Korps zu werben. ${ }^{13}$ In der Annahme, das NSKK auf seiner Seite zu haben, äußerte er sich auf der Tagung des RTÜV am 23./24. Februar $1938 \mathrm{sehr}$ selbstbewusst: „Wir haben die Freundschaft der beiden zuständigen Ministerien (RIM, RVM). Und nunmehr gehört auch das NSKK zu uns."14 Dass diese Einschätzung trügerisch war, zeigte sich in den folgenden Monaten. Mit Schreiben vom 19. Juli 1938 an den „Stellvertreter des Führers“ bat Hühnlein um die Voll-

8 Vgl. Niederschrift über die Beiratssitzung des RTÜV vom 30. 5. 1938, BArch NS 24/111; Hoffmann: Unabhängig und neutral, S. 150f. Zur Bedeutung des Reichskraftwagen-Betriebsverbandes siehe S. 166 dieser Arbeit.

9 Vgl. 100 Jahre Technischer Überwachungs-Verein, Bayern e.V., S. 62.

$10 \mathrm{Vgl}$. Hoffmann: Unabhängig und neutral, S. $151 \mathrm{ff}$.

11 Vgl. ebenda. S. 152. Weitere ministerielle Eingriffe des Reichswirtschaftsministeriums, wie etwa die Zwangsmitgliedschaft von Unternehmen, deren Anlagen überwacht werden mussten, und das Führerprinzip, nach dem der Vorsitzende des jeweiligen Vereins von einem Beirat und dem RWM berufen wurde, brachten den Status der rechtsfähigen Vereine bürgerlichen Rechts ins Wanken, ohne ihn jedoch zu Fall zu bringe; vgl. Wiesenack: Wesen und Geschichte der Technischen Überwachungs-Vereine, S. $87 \mathrm{ff} ., 91$.

12 Vgl. Schreiben des RTÜV an den Korpsführer vom 30. 12. 1937, BArch NS 24/111.

13 Vgl. Schreiben von Mayer an den Inspekteur Technik Kraus vom 13. 5. 1938, ebenda.

14 Meldung von Hösl an Kraus vom 3. 3. 1938 über die Tagung des RTÜV am 23./24. 2. 1934, ebenda. 
macht, im Auftrag der Partei das Sachverständigenwesen zusammenzufassen und „innerlich auszurichten“. ${ }^{15}$ Der Korpsführer begründete sein Anliegen mit dem Eigenleben der Vereine, das trotz der Dienstaufsicht der Behörden zu sehr ausgeprägt sei. Er kritisierte die immer noch zu hohen Prüfgebühren und den Umstand, dass die Vereine an die Interessen der Industrie gebunden seien. Außerdem, so lautete ein ideologischer Vorwurf, fehle den Prüfern die Verbundenheit mit dem ,jungen motorisierten Deutschland“, als dessen Anwalt und Förderer sich das NSKK betrachtete. ${ }^{16}$

Gleichzeitig verstärkte der Korpsführer den Druck auf den RTÜV in der Öffentlichkeit. Er sprach in einem Artikel im Völkischen Beobachter vom 23. Juli 1938 in Bezug auf die Technischen Überwachungsvereine von „überlebten Vereinen, in denen die Kraftfahrsachverständigen heute noch organisiert " seien. ${ }^{17} \mathrm{Au}$ Berdem beanstandete er die unzureichende Prüfung von Fahrlehrern bei den Neuzulassungen durch amtliche Sachverständige und ihre mangelnde gutachterliche Kompetenz in Gerichtsverfahren. ${ }^{18} \mathrm{Zu}$ den fachlichen Streitigkeiten traten atmosphärische Störungen. Es wurde der Korpsführung das Gerücht zugetragen, dass sich Sachverständige in Vereinen abfällig über das NSKK geäußert hätten. ${ }^{19}$

Der RTÜV und die angeschlossenen Überwachungsvereine protestierten gegen die Vorwürfe. Rudolf Rist, Direktor des Bayerischen Revisions-Vereins, verteidigte in einem Schreiben vom 23. August 1938 die Vereinsstruktur und wies Vorwürfe in Bezug auf die fehlende politische Zuverlässigkeit zurück. ${ }^{20}$ Der RTÜV wehrte sich vehement gegen Neugestaltungs- und Übernahmepläne durch das Korps. Mit dieser offensiven Haltung unterschied sich der Vorstand der Technischen Überwachungsvereine von den Automobilclub-Funktionären, die vor ihrer Fusion versucht hatten, das Wohlwollen des Korpsführers durch Kooperation und Anpassung zu erringen. Um zu verhindern, dass sich die Auseinandersetzung zu einem Privatkrieg zwischen dem Korps und dem RTÜV entwickelte, erstellte der Reichsverband Ende August 1938 eine Denkschrift, die er den zuständigen Ministerien, dem "Stellvertreter des Führers" und dem Chef der Reichskanzlei zuleitete. ${ }^{21}$ Darin verwies der RTÜV auf die 70-jährige erfolgreiche Tätigkeit der Technischen Überwachung unter Aufsicht und Lenkung des Staates. Die Vereine

15 Vgl. Schreiben des Korpsführers an den Stellvertreter des Führers vom 19. 7. 1938, betr. Sachverständigenwesen, BArch NS 24/90.

16 Vgl. ebenda. Im Mai 1938 erhob Kraus auf der Beiratssitzung Vorwürfe gegen den RTÜV. Er bemängelte, dass es bei den Vereinen zu viele weltfremde Prüfer gebe, denen das „Herz für die Jugend“ fehle. Die Erziehung der Jugend zur Motorisierung sei aber das Hauptziel; vgl. Niederschrift über die Beiratssitzung am 30.5. 1938 in Stuttgart, BArch NS 24/111.

17 Verbandsführer Mayer zitierte in seinem Schreiben an den Korpsführer vom 1.8.1938 aus dem Völkischen Beobachter, BArch NS 24/111.

18 Vgl. Übersicht über das Sachverständigenwesen im deutschen Verkehrsstrafrecht (geheim), zusammengestellt durch die NSKK-Abteilung Gnadensachen und Verkehrsstrafrecht vom 21. 10. 1937 (gebundenes Manuskript), BArch NS 24/676.

19 Vgl. Schreiben von Inspekteur Kraus an Mayer vom 1. 10. 1938, BArch NS 24/111.

20 Schreiben von Ingenieur Rudolf Rist, Direktor des Bayerischen Revisionsvereins, an den Korpsführer vom 23. 8. 1938, ebenda.

$21 \mathrm{Vgl}$. Schreiben des RTÜV an den Korpsführer vom 7. 9. 1938 und Denkschrift vom 29. 8. 1938 , ebenda. 
seien auch im „Dritten Reich“ die geeigneten Stellen für die technischen Prüfungen, weil „der technisch fortschrittliche, ingenieurmäßige Geist im Überwachungsdienst mit der Selbstverwaltung der Vereine stehe und falle“.22 Den Forderungen des Korpsführers nach Loslösung des Kraftfahrwesens von den Ingenieurkörpern setzte der RTÜV die Überzeugung entgegen, dass es ein Grundsatz der Partei sei, wonach bewährte Organisationen nicht in ihrer Tätigkeit gestört werden sollten. Der RTÜV gab zu bedenken, dass der „organische Aufbau“ des Sachverständigenwesens durch eine Abtrennung des Kraftfahrwesens zerstört werde.

Trotz des massiven Protests von Seiten des RTÜV gab sich Hühnlein optimistisch, den Einfluss des NSKK auf das Kraftfahrzeug-Prüfwesen ausweiten zu können. Er fühlte sich zur Übernahme des Sachverständigenwesens ermutigt, da er die grundsätzliche Zustimmung des Stabsleiters der Dienststelle des „Stellvertreters des Führers" Martin Bormann erhalten hatte und sich durch die persönliche Vollmacht des „Stellvertreters des Führers“ Rudolf Heß vom 10. August 1938 abgesichert wusste. Darin wurde dem Korpsführer die Beobachtung der Kraftfahrzeugwirtschaft und der Kraftfahrsachverständigen im Auftrag der NSDAP anvertraut. ${ }^{23}$

Vor diesem Hintergrund glaubte sich Hühnlein in überlegener Position. In seinem Schreiben vom 14. September 1938 an den RTÜV stellte er mit Nachdruck fest:

„Damit nun bei Ihnen keinerlei Unklarheit mehr besteht, wie ich über das Prüfungswesen in der Motorisierung denke, erkläre ich Ihnen hiermit ausdrücklich, daß ich alle Schritte unternommen habe und auch noch unternehmen werde, um die Prüfung von Kraftfahrzeugen, Kraftfahrzeugführern, Kraftfahrlehrern, Kraftfahrsachverständigen usw. unter eine entsprechende Dienstaufsicht der Partei, und, da ich als Führer des NSKK hier Sachbearbeiter des Stellvertreters des Führers bin, praktisch unter meine Dienstaufsicht zu nehmen und organisch mit der Arbeit des Korps gleichzuschalten. ${ }^{\text {"24 }}$

\section{Die Stellungnabmen der Ministerien}

Verärgert über die Uneinsichtigkeit der Technischen Überwachungsvereine ersuchte Hühnlein um eine Entscheidung an höchster politischer Stelle. Er forderte einen „Führerentscheid“ und knüpfte in seinem Antrag an einen bereits ergangenen Auftrag Hitlers an das NSKK an, alle Omnibusse im Reich auf ihre Betriebssicherheit zu überprüfen. ${ }^{25}$ Bevor die Reichskanzlei das Gesuch des Korpsführers

22 Denkschrift vom 29. 8. 1938, betr. Amtliche Kraftfahrsachverständige, ebenda.

23 Vgl. Schreiben von Martin Bormann an den Korpsführer vom 20. 7. 1938, BArch NS 24/ 90; Der NSKK-Mann vom 22. 10. 1938: „Förderung der Motorisierung“.

24 Schreiben von Hühnlein an den RTÜV-Vorsitzenden Mayer vom 14. 9. 1938, BArch NS 24/111.

25 Vgl. Schreiben des Stellvertreters des Führers an den Chef der Reichskanzlei vom 14. 10. 1938, BArch R 43 II 749a; Vollmacht des Führers an Korpsführer Hühnlein vom 16. 6. 1938; vgl. im Westdeutschen Beobachter vom 16.6. 1938: „Jedes Leben ist kostbar“. Gemäß Erlaß des Reichsverkehrsministers vom 17.6. 1939 wurden die Führerscheinprüfungen für Omnibusfahrer vor einem Prüfungsausschuss abgelegt, dem auch ein NSKK-Führer angehörte; vgl. Mitteilung des Amtes Verkehr vom 27. 6. 1939, BArch NS 24/282, 659. 
an Hitler weiterleitete, sammelte sie die Stellungnahmen der beteiligten Ministerien.

Der argumentationsschwache Schnellschuss des Korpsführers traf auf weitgehende Ablehnung innerhalb der Ministerien. Das Reichsverkehrsministerium sprach sich gegen eine Eingliederung des Sachverständigenwesens in das NSKK aus. Es stellte klar, dass sich der bisherige „Führerauftrag“ nur auf die Nachprüfung der Eignung von Omnibusfahrern, nicht aber auf die Überprüfung der Omnibusse beziehe. Der Reichsverkehrsminister befürchtete, dass eine Übertragung der Prüfungsrechte auf das NSKK eher eine Verschlechterung der Kontrollqualität mit sich bringen würde. Außerdem, so das Ministerium, sei die Unabhängigkeit und Unparteilichkeit der Sachverständigen nicht gewahrt, da dem NSKK Industrievertreter und Inhaber von Reparaturwerkstätten angehörten. ${ }^{26}$ Vor allem aber ergebe sich der „unhaltbare Zustand, daß der Reichsverkehrsminister (als Staat) den Kraftfahrzeugsachverständigen (als Angehörigen einer Parteigliederung) Befehle erteilen müsse".27

Auch das Reichswirtschaftsministerium, das Reichsinnenministerium und der Generalbevollmächtigte der Kriegswirtschaft sprachen sich gegen eine Neuorganisation des Sachverständigenwesens aus. ${ }^{28}$ Konsens herrschte bei den angefragten Ministerien darüber, dass das NSKK die Sachverständigen auf ihre Eignung im fachlichen und weltanschaulichen Bereich überprüfen sollte, wie dies bei den Fahrlehrern bereits geschehe. Der Vorschlag, das Sachverständigenwesen als Ganzes in die NSDAP-Gliederung zu überführen, wurde von den Behörden hingegen verworfen. ${ }^{29}$

Ausschlaggebend für die ablehnende Haltung der Ministerien war die fachlich gute Arbeit der Sachverständigenvereine und die Tatsache, dass die Eingliederung der Sachverständigen in das NSKK verwaltungstechnische Komplikationen und unnötiges Kompetenzgerangel heraufbeschworen hätte. Schwerer noch wog das Argument, der Reichsverkehrsminister und der RTÜV würden im Rahmen der Landesverteidigung Verantwortung für einen großen Bestand an funktionstüchtigen Fahrzeugen tragen - eine wichtige Aufgabe, die nicht gestört werden dürfe. ${ }^{30}$

Der Korpsführer wurde durch die große Geschlossenheit der Ablehnungsfront und die erfolgreiche Lobbyarbeit des Reichsverbandes der Technischen Überwachungsvereine daran gehindert, seine Befugnisse zu erweitern. Verantwortlich für die Niederlage waren vor allem Widerstände im Reichsverkehrsministerium. Das

26 Vgl. Schreiben des Reichsverkehrsministers an den Chef der Reichskanzlei vom 7.11. 1938, BArch R 43 II 749a. Als zusätzliches Gegenargument nannte der RVM noch den unklaren Status, den die Sachverständigen als pensionsberechtigte Angestellte und NSKKAngehörige hätten.

27 Aktenvermerk der Reichskanzlei vom 28. 10. 1938, BArch R 43 II 749a.

$28 \mathrm{Vgl}$. Schreiben des Generalbevollmächtigten für die Kriegswirtschaft an die Reichskanzlei vom 18. 11. 1938, BArch R 43 II 749a, Bl. $25 \mathrm{f}$.

$29 \mathrm{Vgl}$. das Schreiben des Reichsministers des Innern an den Chef der Reichskanzlei vom 28. 2. 1939, BArch R 43 II, 749a Bl. $55 \mathrm{ff}$.

30 Vgl. den Erlaß des Reichsverkehrsministers vom 28. 3. 1938 an den RTÜV gelegentlich der militärischen Vormusterung, in: Denkschrift vom 29.8. 1938, betr. Amtliche Kraftfahrsachverständige, BArch NS 24/111. 
Ministerium befürchtete durch die Einmischung des NSKK den Verlust der Oberhoheit. In seiner Stellungnahme machte Minister Julius Dorpmüller sehr deutlich, dass das NSKK Sand ins Getriebe eines gut funktionierenden Auftragsverhältnisses zwischen TÜV und RVM streue. ${ }^{31}$

Ein im politischen System angelegtes „Prinzip der Kompetenzkonkurrenz“32 wird hier erkennbar. Die mit Einzelaufträgen ausgestattete Parteigliederung versuchte, den bestehenden Behörden und traditionellen Berufsvereinigungen Befugnisse streitig zu machen. Erfolgreich waren solche Unternehmungen im Falle des Beauftragten für den Vierjahresplan, der seit 1936 das Reichswirtschaftsministerium in den Schatten stellte und auch im Falle des Generalbevollmächtigten für den Arbeitseinsatz, der 1942 die Macht des Reichsarbeitsministeriums schmälerte. ${ }^{33}$ Auf der Grundlage von Ernst Fraenkels vorgenommener typologischer Unterscheidung zwischen "Normen- und Maßnahmenstaat“, die das formale Fortbestehen der Reichsverfassung, geschwächt durch die Einflussnahme nationalsozialistischer Organisationen, beschreibt, ${ }^{34}$ lässt sich der Versuch Hühnleins, durch Vollmachten des „Stellvertreters des Führers“ oder Hitlers selbst die Entscheidungsbefugnis des Reichsverkehrsministeriums zu untergraben, erklären. Doch gelang es ihm nicht, eine Bevollmächtigung des „Führers“ zu erhalten, die es ihm ermöglicht hätte, die Interessen und Machtbefugnisse der beteiligten Kräfte zu umgehen. Noch bevor es zum Vortrag bei Hitler kam, zog der Korpsführer am 16. Februar 1939 seinen wenig Erfolg versprechenden Antrag zurück. 35 Er gab sich damit zufrieden, dass die Zulassung amtlich anerkannter Kraftfahrsachverständiger in Zukunft abhängig von der Zustimmung des Korps war und der Nachweis der „arischen“ Abstammung vorgelegt werden musste. ${ }^{36} \mathrm{Als}$ Zuge-

31 Der TÜV hatte nach Meinung von Kraus auch deshalb nichts "gelernt“, weil die Sachbearbeiter des Reichsverkehrsministeriums zu ihm hielten; vgl. das private Schreiben von Kraus an Gustav Fischer vom 17. 1. 1941, BArch NS 24/120.

32 Vgl. Hachtmann: Thesen zur „Modernisierung“ der Industriearbeit, S. 439. Zur Kompetenzzersplitterung in der Verkehrspolitik vgl. Kopper: Modernität oder Scheinmodernität, S. 411.

33 Beispiele hierfür bei Hachtmann: Thesen zur „Modernisierung“ der Industriearbeit, S. 439.

34 Die Bezeichnungen „Maßnahmenstaat“ und „Normenstaat“ als Charakteristika der Herrschaftsstruktur des Nationalsozialismus gehen zurück auf Fraenkel: Der Doppelstaat. Ein Beispiel für die „Entstaatlichung“ des Systems stellt der Machtgewinn der Gauleiter dar; vgl. Mommsen: Nationalsozialismus als vorgetäuschte Modernisierung, S. 409.

35 Hühnlein kritisierte das Reichsverkehrsministerium für die mangelnde Gesprächsbereitschaft und zeigte sich verärgert darüber, dass andere Ministerien einbezogen werden, die „gar nicht zuständig“ seien. Die Rücknahme des Gesuchs begründete Hühnlein aber nicht mit seinen geringen Erfolgsaussichten, sondern mit vermögensrechtlichen Abwicklungsschwierigkeiten der Vereine; vgl. Schreiben des Korpsführers des NSKK an den Reichsminister und Chef der Reichskanzlei Dr. Lammers vom 16.2. 1939, BArch R 43 II 749a, Bl. $46 \mathrm{ff}$.

36 Vgl. die Notiz über die Besprechung am 28. 2. 1939 bei Ministerialdirektor Brandenburg, BArch R 43 II 749a, Bl. 62; Anordnung des Reichsverkehrsministers zur Verordnung über Sachverständige für den Kraftfahrzeugverkehr mit Prüfungsordnung vom 11.1. 1940, BArch NS 24/283. Nicht erst 1939 machte das NSKK seinen Einfluss bei Neuzulassungen von Sachverständigen geltend. Auf die Anfrage der Stuttgarter Handelskammer beim Chef des Kraftfahrwesens der SA nach der Zuverlässigkeit eines Bewerbers sprach sich In- 
ständnis sagte das Reichsverkehrsministerium dem Korpsführer $1940 \mathrm{zu}$, das Thema nach Ende des Krieges noch einmal aufzurollen. ${ }^{37}$ Außerdem hatte die NSKK-Korpsführung sichergestellt, dass seine Sachverständigen als hauptamtliche Angestellte des Korps bereits seit 1936 auf den Motorsportschulen (MSS) weitgehend souverän agierten. ${ }^{38}$

Die Repräsentanten der Technischen Überwachungsvereine erfüllte es mit „tiefer Befriedigung", dass durch die Verordnung über Sachverständige für den Kraftfahrzeugverkehr vom 6. Januar 1940 die Verdienste des RTÜV auf dem Gebiet des Kraftfahrwesens Anerkennung fanden. ${ }^{39}$ Die Prüfstellen der Vereine dienten auch weiterhin als Träger der technischen Überwachung des Kraftfahrzeugverkehrs.

\section{Die Entwicklung der Fahrschulausbildung und der Fahrlehrerverbände}

Die Förderung der Motorisierung ließ sich nicht ausschließlich durch eine Erhöhung der Fahrzeugdichte im Deutschen Reich erreichen, sondern gleichermaßen über eine Steigerung der Zahl an Führerscheinbesitzern. Um diesem Ziel näher zu kommen, plante das Reichsverkehrsministerium als Impulsgeber im gesetzgeberischen Bereich einen vereinfachten und kostengünstigen Zugang zum Führerschein. Bis 1933 übernahmen rund 1000 konzessionierte Fahrschulen die obligatorische Ausbildung der Führerscheinbewerber. ${ }^{40}$ Die Fahrschulen wandten sich gegen eine in der Öffentlichkeit diskutierte Aufhebung der Ausbildungspflicht. Sie sahen ihre wirtschaftlichen Interessen gefährdet und befürchteten eine Steigerung der Unfallzahlen. ${ }^{41}$ Auch die nationalsozialistische Presse ${ }^{42}$ und das NSKK

spekteur Technik Kraus gegen die Anerkennung des Kandidaten aus. Dieser sei als Gegner der NSDAP bekannt; vgl. Schreiben der Handelskammer Stuttgart an den Chef des Kraftfahrwesens der SA vom 29. 3. 1934 und die Antwort von IT Kraus an die Handelskammer vom 5. 4. 1934, BArch NS 24/250.

37 Vgl. das Schreiben des Korpsführers an den Führer der Motorgruppe Thüringen, Gruppenführer Barth, vom 2. 8. 1941, BArch NS 24/142. Hühnlein war mit den vor dem Krieg getroffenen Regelungen unzufrieden; vgl. den Bericht zur Frage der Betätigung der Technischen Überwachungsvereine auf dem Gebiet des Kfz-Wesens, 1940 oder später, BArch NS 24/313.

38 Vgl. Schreiben des Korpsführers des NSKK an den Reichsminister und Chef der Reichskanzlei Dr. Lammers vom 16. 2. 1939, BArch R 43 II 749a, Bl. $46 \mathrm{ff}$.

39 Vgl. Technische Überwachung vom 16. 3. 1940: „Die Technische Überwachung des Kraftfahrzeugverkehrs".

40 Die Automobilclubs bezeichneten die Fahrschulen als Feinde des Automobilismus und forderten die Abschaffung des „Fahrschulzwangs“; vgl. Schreiben des Reichsverbandes Deutscher Kraftfahrschulen an den Regierungspräsidenten von Potsdam vom 3.3. 1933, BLHA Potsdam, Rep. 2 A, Regierung Potsdam Abt. IV Nr. 1364.

41 Vgl. Denkschrift des Reichsverbandes Deutscher Kraftfahr- und Fliegerschulen zur Frage einer geplanten Aufhebung des Fahrschulzwanges vom 25.7.1933 und Eingabe des Verbandes an die Reichsregierung vom 9. 9. 1933, BLHA Potsdam, Rep. 2 A, Regierung Potsdam Abt. IV Nr. 1364; Denkschrift „Zwingende Erfordernisse der Kraftverkehrswirtschaft im nationalsozialistischen Staate“ vom Reichsbund der Kraftverkehrs-Technik 
traten für den Fortbestand einer geregelten Ausbildung ein. Der Korpsführer machte in seiner programmatischen Rede anlässlich der Flaggenweihe des DDAC am 30. November klar: „Wir treten ein für den Beibehalt des Ausbildungszwanges und behördlichen Prüfungszwanges. Der streng geprüfte und gewissenhaft zugelassene Fahrlehrer ist hierbei der verantwortliche Träger der Ausbildung, nicht der Besitzer der Fahrschule." 43

Das Fahrschulwesen wurde in diesem Sinne reformiert. Die Verordnung über die Ausbildung von Kraftfahrzeugführern vom 21. Dezember 1933 beseitigte das Monopol der Fahrschulen. ${ }^{44}$ Das Recht zur Ausbildung ging von den Fahrschulen auf jeden behördlich geprüften Fahrlehrer über. ${ }^{45}$ Der Fahrlehrer musste behördlich geprüft, nicht aber besonders ausgebildet sein, da sich nach Meinung des Reichsverkehrsministers das Anerkennungsverfahren für Fahrlehrer ohnehin zu einem reinen "Attesthandel" über die medizinische Eignung entwickelt hatte.46 Außerdem war die Zulassung des Fahrlehrers nicht mehr von einer dreijährigen Fahrpraxis und dem behördlich festgelegten Bedarf an Ausbildern oder dem Nachweis bestimmter Lehrmittel abhängig. ${ }^{47}$ Falls zur Ausbildung keine öffentlichen Wege benutzt wurden, entfiel für den Führerscheinbewerber sogar die Notwendigkeit, einen behördlich zugelassenen Fahrlehrer aufzusuchen. ${ }^{48}$ Weitere Lockerungen, die den Führerscheinerwerb vereinfachten, waren der Wegfall der Mindestausbildungsdauer und die amtsärztliche Untersuchung für Fahrschüler. ${ }^{49}$

Diese freizügige Handhabung der Ausbildung von Fahrschülern entsprach dem Motorisierungswillen des Regimes. Um eine schnellere Erhöhung der Zahl an Führerscheininhabern in Deutschland zu erreichen, wandte das Reichsverkehrsministerium Strategien an, die bereits aus der zeitgenössischen Perspektive heraus kurzsichtig genannt werden können. Die gesetzlichen Erleichterungen, die im „Interesse der Motorisierung Deutschlands“ eingeführt worden waren, hatten in

(Eingang der Denkschrift in Potsdam am 25. 6. 1933), BLHA Potsdam, Rep. 2 A, Regierung Potsdam Abt. IV Nr. 1364.

42 Vgl. Sonderdruck aus dem Völkischen Beobachter, Beiblatt Berliner Beobachter vom 29. 9. 1933: „Sabotage der Arbeitsförderung“.

43 Amtliche Mitteilungen des DDAC vom 1. 12. 1933: „Richtfest und Flaggenweihe im DDAC“.

44 Vgl. Das Auto und Kraftrad vom Februar 1935: „Reichsverband der Kraftfahrlehrer“; „Verordnung über die Ausbildung von Kraftfahrzeugführern vom 21. 12. 1933“, RGBl. I 1934, Nr. 2.

45 Vgl. Abschrift des Schreibens des Reichsverkehrsministers an den Preußischen Minister für Wirtschaft und Arbeit vom 18. 1. 1934, StA Münster, Regierung Münster Nr. 8774.

46 Vgl. Der deutsche Volkswirt, (Kraftverkehrswesen im neuen Deutschland, Sonder-Beilage) vom 2. 3. 1934: „Was kann der Staat für die Motorisierung des Verkehrs tun?" von RVM von Eltz-Rübenach.

47 Vgl. Kraftfahrt-Pressedienst vom 28. 9. 1934: „Fahrschüler und Fahrlehrer“; Schreiben des Reichs- und Preußischen Verkehrsministers an die obersten Landesbehörden vom 15. 5. 1935, StA Augsburg, Bezirksamt Krumbach Nr. 730.

48 Vgl. Verkehrswarte vom Mai 1934: „Führerschein ohne Fahrlehrer“; Fack: Das deutsche Kraftfahrschulwesen, S. 127.

49 Vgl. Kraftfahrt-Pressedienst vom 28. 9. 1934: „Fahrschüler und Fahrlehrer“. 
vielerlei Hinsicht nachteilige Konsequenzen. ${ }^{50}$ Zahlreiche Neugenehmigungen von Fahrlehrern auf der Grundlage der neuen Verordnung zur Ausbildung von Kraftfahrzeugführern waren die Folge. Durch die verschärfte Konkurrenzsituation unter den Fahrlehrern und den Wegfall behördlich festgesetzter Ausbildungsgebühren verringerten sich die Kosten für die Ausbildung. ${ }^{51}$ Dies kam den Führerscheinanwärtern entgegen, schmälerte aber andererseits die Einnahmen der Fahrlehrer und gefährdete ihre wirtschaftliche Existenz. Eine weitere negative Konsequenz, die durch den Wegfall einer gesetzlich geforderten Ausbildung der Kraftfahrlehrer und einer nicht näher spezifizierten Ausbildung für Führerscheinbewerber eintrat, war die Verschlechterung der Qualitätsstandards der Ausbildung. Dies musste sich negativ auf die Fahrtüchtigkeit bei Fahranfängern auswirken und stellte eine erhebliche Gefahr für die Verkehrssicherheit in Deutschland dar. ${ }^{52}$

\section{Organisatorische Entwicklung}

Die in verschiedenen Fahrlehrerverbänden organisierten Fahrlehrer waren sich bewusst, dass nur eine geschlossene Haltung und ein gemeinsamer Verband ihre Interessen wirkungsvoll vertreten konnte. Sie erhofften sich 1934 noch die Unterstützung des Korpsführers:

„Nur wenn es uns gelingt, im neuen Verband alle Fahrlehrer zu erfassen und sie dem Chef des Kraftfahrwesens zu unterstellen, können wir darauf rechnen, daß - entgegen der Verordnung vom 21. Dezember 1933 - die Bedürfnisfrage in Zukunft trotzdem geprüft, der Fahrlehrerberuf vor zweckloser Überfüllung geschützt und die Ausbildung der Kraftfahrer in Deutschland auf der bisherigen hohen Stufe erhalten wird." 53

Kurzzeitig stellte das NSKK selbst Überlegungen an, das Fahrlehrergewerbe institutionell an das Korps zu binden. In der Konsequenz hätte dies die Abschaffung des Fahrlehrerberufs als freien Berufsstand bedeutet. ${ }^{54}$ Die Übernahmepläne wurden jedoch verworfen, weil die Ausbildung von Zivilpersonen in keiner Weise dem Aufgabengebiet des Korps entsprach. Die Korpsführung, die einem Machtgewinn auf dem Gebiet des Kraftfahrwesens niemals abgeneigt war, schreckte da-

$50 \mathrm{Vgl}$. Schreiben des Reichs- und Preußischen Verkehrsministers an die obersten Landesbehörden vom 15. 5. 1935, StA Augsburg, Bezirksamt Krumbach Nr. 730.

51 Eine neue Gebührenordnung wurde in Aussicht gestellt, wenn die Fahrlehrer im Zuge des berufsständischen Aufbaus organisiert seien; vgl. Der Preussische Minister für Wirtschaft und Arbeit vom Januar 1934, GStA PK Berlin, CVIII 1 Nr. 119, Bd. 5.

52 Die Einschätzung trug auch der Führer der Motorgruppe Thüringen in seinem Schreiben an den Korpsführer vom 20.1.1941 vor: „Die hohen Unfallzahlen nehmen in Anbetracht der unfähigen Fahrlehrer nicht wunder“; BArch NS 24/750. Vgl. dazu das Kapitel „Das Ideal: Verkehrssicherheit auf den Straßen des ,Dritten Reiches"“ dieser Arbeit.

53 Rundschreiben des Reichsverbandes deutscher Kraftfahrschulen, des Verbandes der Inhaber privater Kraftfahrkurse Bayerns, der Vereinigung der Fahrlehrer Bayerns an alle derzeitigen Inhaber des Fahrlehrerscheins vom 14. 1. 1934, HStA Stuttgart, E 151/07/Bü 127.

54 Überlegungen, die Fahrerausbildung im NSKK zu übernehmen, kursierten auch als Gerücht im NSKK; vgl. G. L. Wagener: Warum sollte ich verschweigen, S. 649, StadtA Hannover. 
vor zurück, ein „Erwerbsunternehmen“ zu werden, das nach eigenen Aussagen dadurch "viel Verdruß und Behördenkram" bekommen hätte. 55

Im Zuge der nationalsozialistischen "Gleichschaltung" und der Bemühungen für einen organischen Aufbau der Wirtschaft rief der Reichsverkehrsminister am 13. Dezember 1934 eine einheitliche Berufsorganisation der Kraftfahrlehrer, den „Reichsverband der Kraftfahrlehrer“, ins Leben. Alle Fahrlehrer mussten die Mitgliedschaft erwerben. Am 1. Oktober 1935 wurde der Reichsverband in „Fachgruppe Kraftfahrlehrer" umbenannt und in die Reichsverkehrsgruppe Kraftfahrgewerbe eingegliedert. ${ }^{56}$ Bis zum 1 . Oktober 1936 erwarben 7200 Fahrlehrer die Mitgliedschaft der Fachgruppe. Damit hatte sich die Zahl der Fahrlehrer seit Erlass der neuen Ausbildungsvorschrift am 21. Dezember 1933 verdreifacht. Nicht mitgezählt waren hierbei die Ausbilder des Reichsheers, der Reichsbahn und der NSKK-Motorsportschulen. ${ }^{57}$

Der Öffnung des Fahrlehrergewerbes, die zu einer regelrechten „Fahrlehrerinflation" geführt hatte, begegneten die Behörden mit einer Fahrlehrersperre am 31. Januar 1935. ${ }^{58}$ Der gleiche planlose Aktionismus, der die Verantwortlichen im Reichsverkehrsministerium 1933 dazu veranlasst hatte, den Zugang zum Fahrlehrergewerbe zu öffnen und Ausbildungsvorschriften für den Führerscheinerwerb abzuschaffen, ohne die möglichen Konsequenzen zu bedenken, veranlasste das Ministerium ein Jahr später, die "Schotten" wieder dicht zu machen. Auch die Aufhebung behördlich festgelegter Ausbildungstarife wurde rückgängig gemacht, indem die Fachgruppe am 20. Juli 1936 wieder eine Preisordnung erließ ${ }^{59}$

Die radikale Abriegelung des Fahrlehrerberufs war ebenfalls nicht von Dauer. Am 20. September 1935 lockerten die Behörden die Fahrlehrersperre, so dass die höheren Verwaltungsbehörden dort, wo Fahrlehrer fehlten, neue Fahrlehrer zulassen konnten. ${ }^{0}$ Damit wurde die Zulassung wieder von der Bedarfsfrage abhängig gemacht, die bereits vor 1933 mitbestimmend gewesen war.

55 Der genaue Zeitpunkt und der Verlauf der Diskussion sind nicht bekannt. Kraus rekapitulierte in seinem Schreiben an Hühnlein vom 2. 8. 1941 den zurückliegenden Streit um das Fahrlehrergewerbe; BArch NS 24/142.

56 Schreiben des Reichs- und Preußischen Verkehrsministers vom 25. 9. 1935 über die Überführung des Reichsverbandes in die Reichsverkehrsgruppe Kraftfahrgewerbe vom 1. 10. 1935, abgedruckt in: Mitteilungen des Reichsverbandes der Kraftfahrlehrer e. V.,1. Jg. 1935, Nr. 6.

57 Der gewerbliche Kraftverkehr vom 23. 11. 1936: „Der Kraftfahrlehrerberuf im Rahmen des Motorisierungsprogramms".

58 Vgl. Schreiben der Reichsverkehrsgruppe Kraftfahrgewerbe, Fachgruppe Kraftfahrlehrer, Bezirksfachgruppe Bayern an das NSKK vom 27. 12. 1937, BArch NS 24/117.

59 Vgl. Der gewerbliche Kraftverkehr vom 23. 11. 1936: „Der Kraftfahrlehrerberuf im Rahmen des Motorisierungsprogramms" von Kleist, dem Leiter der Fachgruppe Kraftfahrlehrer.

60 Vg. ebenda. Zur Fahrlehrerprüfung traten 1936: 829, 1937: 561 und 1938: 489 Fahrlehrer an; Reichsverband der deutschen Automobilindustrie (Hrsg.): Tatsachen und Zahlen der Kraftverkehrswirtschaft 1937, S. 152 und 1938, S. 117. 


\section{Weltanschauliche Überwachung der Fahrlehrer und marktwirtschaftliche Eingriffe}

Auch wenn es nicht zu einem organisatorischen Anschluss der Fahrlehrer an das NSKK kam, beharrte die Korpsführung des NSKK doch auf einer Einflussnahme in diesem Bereich. Da der Fahrlehrerberuf erzieherische Aufgaben beinhaltete und der Korpsführer an der Schaffung eines "neuen Verkehrsmenschen“61 mitwirken wollte, beanspruchte das NSKK die Aufsicht über die Fahrlehrer sowie die Auswahl und Prüfung der Fahrlehrer. ${ }^{62}$ Der Reichsverkehrsminister kam diesem Ansinnen teilweise entgegen, indem er mit dem Erlass vom 16. Dezember 1937 das NSKK anwies, eine Nachprüfung aller Fahrlehrer im Deutschen Reich vorzunehmen. ${ }^{63} \mathrm{Da}$ das Korps aus ideologischen Gründen die Überprüfung jüdischer Kraftfahrlehrer verweigerte, gab der Reichsverkehrsminister diese Aufgabe im September 1938 an die Polizei weiter. 64

Das NSKK legte an die zu prüfenden Fahrlehrer verschiedene Prüfkriterien an. Wichtig waren die persönliche Eignung des Fahrlehrers, die fachliche Kompetenz und das Vorhandensein von Lehreinrichtungen. Die mit der Nachprüfung beauftragten Führer der Motorgruppen forderten neben den Akten der Höheren Verwaltungsbehörden zusätzlich noch persönliche Unterlagen zur Person des Fahrlehrers an - darunter das Zeugnis der örtlich zuständigen Kreisleitung der NSDAP über die politische Zuverlässigkeit. ${ }^{65}$ Befanden die Führer der Motorgruppen den Fahrlehrer für ungeeignet, reichten sie den Vorgang zur Prüfung an den Inspekteur für technische Ausbildung und Geräte im NSKK Erwin Kraus weiter, der den Entzug der Ausbildungserlaubnis veranlasste. 66 Kraus entschied gemäß der Devise: „Unsere Aufgabe ist es, die Fahrlehrer zu erziehen und zu führen, unlautere und unfähige Elemente auszumerzen [...]." ${ }^{47}$ Die Tatsache, dass einer Parteigliederung und nicht der Polizei oder den Technischen Überwachungsvereinen diese Aufgabe übertragen wurde, ist ein Beleg für das Eindringen welt-

61 Zur Definition des „deutschen Verkehrsmenschen“ vgl. S. 382 dieser Arbeit.

62 Vgl. Erlaß des Korpsführers vom 21. 12. 1943, betr. Kraftfahrtechnik-Ausbildung, abgedruckt in: Kraftfahrtechnische Mitteilungen vom 1. 1. 1944, BArch NSD 42/5.

63 Vgl. Erlaß des Reichsverkehrsministers vom 27. 3. 1939, betr. Prüfung der Kraftfahrlehrer, BArch NS 14/103. Die Fahrlehrerüberprüfungen wurden nach der Angliederung Österreichs und des Sudetenlandes auf diese Gebiete ausgeweitet; vgl. BArch NS 24/118.

64 Vgl. Mitteilung des Inspekteurs Technik vom 13. 10. 1938, BArch NS 24/281. Im Regierungsbezirk Frankfurt (Oder) gab es unter den rund 160 Fahrlehrern zwei jüdische Fahrlehrer, die nicht vom NSKK überprüft worden waren; Information der NSKK-Gruppe Kurmark an den Regierungspräsidenten von Frankfurt (Oder) vom 1.12. 1938 und vom 2. 3. 1939, BLHA Potsdam, Rep. 3 B, Regierung Frankfurt Abt. IV Nr. 117. Zum Umgang des NSKK mit jüdischen Fahrlehrern vgl. auch S. 411 und S. 413 f. dieser Arbeit.

$65 \mathrm{Vgl}$. Verordnung des Korpsführers vom 15. 2. 1938, betr. Nachprüfung der Kraftfahrlehrer (Entwurf), BArch NS 24/94.

66 Vgl. Verfügung des Korpsführers vom 19. 1. 1938, BArch NS 24/155.

67 Schreiben von Kraus an Hühnlein vom 2. 8. 1941, BArch NS 24/142. In einer Zwischenfeststellung von 1938 hatte Kraus sogar $50 \%$ aller bereits überprüften Fahrlehrer als unbrauchbar bezeichnet; vgl. Inspekteur Technik an den Korpsführer vom 31. 8. 1938, Auswertung der Halbjahresberichte 1. 4. 1938 bis 30. 9. 1938, BArch NS 24/100. 
anschaulicher Elemente in den Überwachungsapparat des Kraftfahrwesens. Bis zum Kriegsausbruch hatte das Korps nach eigenen Angaben etwa einem Drittel der damals zugelassenen Fahrlehrer die Ausbildungserlaubnis entzogen. Die verbliebenen Fahrlehrer wurden auch im Krieg laufend überwacht. ${ }^{68}$ Die Gründe für die Entziehung der Fahrlehrerscheine lagen meist in den unzulänglichen Lehrräumen und der geringen Lehrbefähigung der Ausbilder. Bei einigen waren Zweifel an der "persönlichen Zuverlässigkeit" ausschlaggebend. ${ }^{69}$

Die Nachprüfung der Fahrlehrer ab Ende 1937 hatte einen Boom von Beitrittsanträgen zum NSKK zur Folge. Viele Fahrlehrer versuchten offenbar, sich durch politische Anpassung berufliche Vorteile zu verschaffen, oder wollten zumindest sicherstellen, keine beruflichen Nachteile zu erleiden. Dies rief beim Korpsführer Zweifel an der Ernsthaftigkeit der Gesuche und der Dienstbereitschaft der eintrittswilligen Fahrlehrer hervor. Deshalb bekräftigte er: „Ich will auf jeden Fall vermeiden, dass hier Politik und Geschäft verquickt werden und sich bestimmte Berufsschichten nur deshalb zeitlich vorübergehend oder auf längere Dauer dem Korps einreihen, weil sie dann dessen Schutz genießen."70

Die Absichtserklärung des Korpsführers war ein Widerspruch in sich, da gerade die Einflussnahme des NSKK ein Beispiel für das Eindringen der Politik in geschäftliche Dinge darstellte; des Weiteren war das NSKK aus pragmatischen Gründen darauf angewiesen, möglichst viele Fahrlehrer anzuwerben, da es von deren Übungsräumen und Fachkenntnissen profitieren wollte.

Die Eingriffe des NSKK in das Kraftfahrlehrergewerbe erstreckten sich nicht nur auf die Nachprüfung der bestehenden Fahrschulen, sondern auch auf das Zulassungsverfahren neuer Fahrlehrer. ${ }^{71}$ Vor Erteilung der neuen Ausbildungserlaubnis baten die Verwaltungsbehörden die zuständigen Motorgruppen um ihre Einschätzung. ${ }^{72}$ Die NSKK-Führer befürworteten die Gesuche dann besonders bereitwillig, wenn der Bewerber Angehöriger des NSKK war und auf die Gutach-

68 Vgl. Schreiben von Kraus an Hühnlein vom 2. 8. 1941, BArch NS 24/142.

$69 \mathrm{Vgl}$. Schreiben der Motorgruppe Brandenburg an die Reg. Frankfurt (Oder) vom 29. 3. 1939, BLHA Potsdam, Rep. 3 B, Regierung Frankfurt IV Nr. 117. Der Inspekteur Technik war bei einer Nachprüfung von 50 Fahrlehrern in Berlin anwesend. Die Mehrzahl habe die technisch notwendigen Berufskenntnisse nicht besessen, so das Urteil; vgl. Meldung des Inspekteurs Technik an Hühnlein vom 27. 5. 1938, BArch NS 24/675.

70 Anweisung des Korpsführers an die Motorgruppen vom 5.11. 1938, (Entwurf), BArch NS 24/93. Nach dem persönlichen Eindruck des NSKK-Gruppenführers von Hannover Georg Wagener hingegen stand der größte Teil der Fahrlehrer im „Dritten Reich“ abseits des NSKK; G. L. Wagener: Warum sollte ich verschweigen, S. 649, StadtA Hannover.

$71 \mathrm{Vgl}$. Anordnung des Inspekteurs Technik an die Motorobergruppen und Motorgruppen vom 24.3. 1939 (Entwurf), betr. Nachprüfung der Kraftfahrlehrer durch das NSKK, BArch NS 24/95.

72 Der Beauftragte der Motorgruppe prüfte auf Anweisung der höheren Verwaltungsbehörden: 1. Charakter und politische Unbedenklichkeit der Person; 2. Fachliche Kenntnisse; 3. Vorhandensein ausreichender Lehrmittel; vgl. Briefbucheintrag des Inspekteurs Technik vom 7. 11. 1938, betr. Verfahren bei Neuzulassung von Fahrlehrern und Runderlaß des Reichsverkehrsministeriums vom 16. 12. 1937, BArch NS 24/281. Vgl. auch das Schreiben der NSKK-Motorgruppe Kurmark an den Regierungspräsidenten Frankfurt (Oder) vom 5. 7. 1938, BLHA Potsdam, Rep. 3 B, Regierung Frankfurt Abt. IV Nr. 117. 
ter einen "weltanschaulich gefestigten“ Eindruck machte. ${ }^{73}$ Anfang des Jahres 1939 wurde eine zentrale Kommission eingerichtet, die sich unter Mitwirkung des NSKK künftig mit der Zulassung neuer Fahrlehrer beschäftigen sollte. ${ }^{74}$

Weltanschauliche Regimetreue wurde zu einem wichtigen Kriterium für die amtliche Ausstellung des Fahrlehrerscheins. Es gelang dem NSKK im Verkehrssektor, in den Bereich herkömmlicher rechtlicher Normen Elemente des „Maßnahmenstaates" einzufügen, die seine Etablierung im öffentlichen Kraftfahrwesen förderten und der Bekämpfung nationalsozialistischer Gegner dienten.

NSKK-Korpsführer Hühnlein intensivierte die Beziehungen zur Fachgruppe Kraftfahrlehrer. In einer Vereinbarung vom 4. Februar 1939 mit dem Fachgruppenleiter Kleist wurde eine für das NSKK vorteilhafte Zusammenarbeit festgeschrieben. Die Fachgruppe verpflichtete sich, sämtliche von ihr erfassten Kraftfahrlehrer zum Eintritt und zur Mitarbeit im Korps aufzufordern. Außerdem fand die Sonderausbildung der Bezirksfachgruppenleiter und aller Lehrkräfte für die Weiterbildung der Kraftfahrlehrer auf der Technischen Führerschule in München statt. Dadurch war es den Fahrlehrern kaum möglich, sich der Aufsicht und Kontrolle durch das NSKK zu entziehen. Als äußeres Zeichen der Zusammenarbeit traten der Leiter der Fachgruppe Kleist und der Geschäftsführer Pagenstecher dem Stab des Korpsführers bei; die Bezirksfachgruppenleiter gliederten sich als ehrenamtliche Referenten den Stäben der NSKK-Motorgruppen an. ${ }^{75}$

Resümiert man die äußeren Einflüsse auf das Fahrlehrergewerbe zwischen 1933 und 1939, ist abgesehen von der Einmischung des NSKK, die von vielen Fahrlehrern als Anmaßung empfunden worden sein muss, das äußerst widersprüchliche politische und bürokratische Vorgehen hervorzuheben. Die angeordnete Öffnung des Fahrlehrergewerbes führte zu einer hohen Zahl an Fahrlehrern und erhöhte die Gefahr der sinkenden Qualität der Ausbildung. Um diese negative Tendenz abzuwehren, beauftragte das Reichsverkehrsministerium das NSKK mit der Nachprüfung der Fahrlehrer. Dies führte zu der eigentümlichen Konstellation, dass hier Elemente des „Maßnahmenstaates“ genutzt wurden, um Fehler des „Normenstaates“ zu korrigieren.

\section{Eingriffe in den freien Markt}

Das durch die schlechte wirtschaftliche Situation Anfang der dreißiger Jahre in die Krise geratene Fahrlehrergewerbe erfuhr ab 1934 durch die Ausbildungslehrgänge des NSKK auf den Motorsportschulen Konkurrenz. Um eine wirtschaftliche Schädigung der privaten Fahrschulen zu vermeiden, verpflichtete sich die Korpsführung am 23. Februar 1935, auf den Motorsportschulen Führerscheine nur für

$73 \mathrm{Vgl}$. Befürwortungsschreiben der NSKK-Motorstandarte 66 Münster vom 16. 2. 1939 an den Regierungspräsidenten in Münster, StA Münster, Regierung Münster Nr. 8667.

74 Vgl. Notiz über die Besprechung am 28. 2. 1939 bei Ministerialdirektor Brandenburg, BArch R 43 II 749a, Bl. 62.

75 Vgl. Krafthand vom 11. 2. 1939: „Arbeitsgemeinschaft NSKK - Kraftfahrlehrer“; Anordnung des Inspekteurs Technik vom 20. 2. 1939, betr. Kraftfahrlehrer, BArch NS 24/94. 
Lehrgangsteilnehmer zwischen 18 und 25 Jahren zu beantragen. ${ }^{76}$ Zur weiteren Besänftigung führten NSKK-Führer an, dass die MSS des NSKK nur die vormilitärische Ausbildung derjenigen Deutschen durchführten, die zu motorisierten Truppenteilen der Wehrmacht gemustert waren. ${ }^{77}$ Die Beruhigungsstrategie, nach der "nur" Rekruten ausgebildet würden und sich diese Zielgruppe die gewerbliche Ausbildung und Prüfung ohnehin nicht leisten könne, vermochte nicht zu überzeugen, denn die Absolventen der Motorsportschulen schieden zu Tausenden auch für die Zukunft als potenzielle Fahrschul-Kunden aus.

Eine ganz persönliche Konkurrenz erwuchs den Fahrlehrern durch die NSKKFahrlehrer der MSS. Die Reichsmotorsportschule in Döberitz bildete eigene Fahrlehrer für das NSKK aus, die danach auf den 24 Motorsportschulen im Reich als Ausbilder tätig waren. Auch hier wurde auf die Interessen der "allgemeinen“ Fahrlehrer Rücksicht genommen. Der Erlass des Reichsverkehrsministeriums vom 5. März 1933 genehmigte die Zulassung von 18 Fahrlehrern pro Motorsportschule mit der Auflage, dass die Fahrlehrer nach ihrem Ausscheiden den Fahrlehrerschein zurückgeben. ${ }^{78}$

Da die Motorisierung in der NS-Zeit eng an die Wehrhaftmachung der Nation geknüpft war, brachte das von Hitler angekündigte Motorisierungsprogramm Nachteile für den Fahrlehrerberuf. Die militärische Form der Motorisierung hatte Priorität vor einer zivilen Förderung der Motorisierung. Ein Protest der Fahrlehrer wäre aussichtslos gewesen, denn auch das Reichsverkehrsministerium gestand dem NSKK verkehrsrechtliche Freiräume auf den MSS zu und gab den militärischen Interessen mehr Gewicht als den beruflichen Existenzängsten der Fahrlehrer. $^{79}$

Der parteigelenkte und staatlich geschützte Ausbildungs- und Beschäftigungssektor, der sich auf den MSS entwickelt hatte, ließ sich zum Bedauern der Korpsführung nicht auf die Basis der Motorstürme und der Motor-HJ übertragen. Die Motorsturmführer wandten sich wiederholt an die Führer der Motorbrigaden und -gruppen mit der Bitte, kostenlose oder preisreduzierte Fahrschulausbildung durch Fahrlehrer in ihren lokalen Stürmen durchführen lassen zu dürfen. ${ }^{80}$ Hühnlein untersagte sturminterne Fahrkurse, denn es vertrage „sich nicht mit der nationalsozialistischen Weltanschauung, das Fahrlehrergewerbe auf diese Weise zu schädigen" ${ }^{81}$ Obgleich nach der NS-Machtübernahme einige Regierungsbe-

76 Vgl. Das Auto und Kraftrad vom 1. 9. 1935: „Führer- und Fahrlehrerscheine für Motorsportschulen".

77 Vgl. Schreiben des Inspekteurs Technik an die NSDAP, Stab des Stellvertreters des Führers vom 14. 11. 1936, BArch NS 24/114; Krafthand vom 25. 7. 1935: „Kraftfahrer-Ausbildung".

78 Vgl. Erlaß des Reichsverkehrsministers vom 5. 3. 1933, in: Das Auto und Kraftrad vom 1. 9. 1935: „Führer- und Fahrlehrerscheine für Motorsportschulen“.

79 Vgl. Abschrift des Erlaßes des Reichsverkehrsministers vom 11. 5. 1935, betr. Kraftfahrlehrer an den NSKK-Motorsportschulen, BArch NS 24/317. Die Genehmigung erfolgte im "wehrpolitischen Interesse", da ein großer Bedarf an Fahrlehrern für die Motorsportschulen bestand.

80 Vgl. Schreiben der Motorstandarte 112 an die Motorbrigade Hansa vom 19.7. 1935, BArch NS 24/136.

81 Vgl. Korpsführung an die Motorbrigade Hessen vom 25. 7. 1935, ebenda. 
zirke und zuständige Revisionsvereine Motor-SA-Stürmen bis zu 50\% Ermäßigung beim Führerscheinerwerb zugestanden hatten, ${ }^{82}$ waren nach Festsetzung der Gebührenordnung 1936 Nachlässe für die Ausbildung von NSKK-Angehörigen gesetzlich verboten. ${ }^{83}$

Doch es kam vor, dass sich insbesondere bei der Motor-HJ die Schulung nah an der Grenze dessen bewegte, was man als Ausbildung von Fahrzeugführern bezeichnen könnte. Fahrlehrer, die in NSKK-Stürmen Dienst taten und auch für die Betreuung der Motor-HJ zuständig waren, kamen in Konflikt mit ihren eigenen Berufsinteressen. Die Kompromissformel lautete, dass kein praktischer Fahrunterricht vorgesehen sei und die Jungen höchstens Beifahrer sein sollten. ${ }^{84}$

Trotz der öffentlichen Beschwichtigungen an die Adresse der Fahrlehrer stand die Motorisierung der eigenen Formation an oberster Stelle in der Wunschliste des Korpsführers. Er versuchte im Laufe des Jahres 1937 den Reichsverkehrsminister davon zu überzeugen, dass eine systematische Ausbildung innerhalb der Breitengliederung des NSKK vonnöten sei. Die Männer sollten nach einer Ausbildung im Sturm nur noch die erforderliche Prüfung vor dem Kraftfahrlehrer und dem amtlichen Sachverständigen ablegen. ${ }^{85}$ Anders als auf den MSS wurden dem NSKK innerhalb seiner Motorstürme keine Sonderkonditionen und keine verkehrsrechtliche Autonomie zugebilligt. Eine Art Entgegenkommen bewies der Reichsverkehrsminister bei der Einführung der Führerscheinklasse 4 für Kleinkrafträder, die am 1. Oktober 1938 in Kraft trat. Das NSKK erhielt die Ermächtigung, seine Mitglieder und Angehörige der $\mathrm{HJ}$ ab dem vollendeten 16. Lebensjahr auf die Kenntnisse der Verkehrsvorschriften selbst zu prüfen. ${ }^{86}$

\section{Grenzen und Möglichkeiten der Einflussnabme des NSKK}

Die organisatorische Neuordnung im Technischen Überwachungswesen hatte positive Modernisierungseffekte zur Folge. Gegen den Widerstand des Allgemeinen Verbandes der Deutschen Dampfkessel-Überwachungsvereine setzte das Reichsverkehrsministerium die Vereinheitlichung der Organisation und der Prüfstrukturen sowie die Schaffung einer Dachorganisation durch. Auf diese Weise

82 Vgl. StA Augsburg, Regierung von Schwaben und Neuburg Nr. 10047. Auch die Regierung von Ober- und Mittelfranken genehmigte die verbilligte Ausbildung für Motor-SAStürme; StA Nürnberg, Regierung von Ober- und Mittelfranken, KdI Abgabe 1978 Nr. 4214.

83 Vgl. Schreiben der Kraftverkehrsgruppe Kraftfahrgewerbe, Fachgruppe Kraftfahrlehrer, Bezirksleitung Südwestdeutschland vom 20. 2. 1937 an einen Fahrlehrer mit der Warnung, er mache sich strafbar, wenn er Angehörigen des NSKK einen Nachlass von $50 \%$ auf die vorgeschriebenen Richtpreise für Ausbildungen gewähre; StA Ludwigsburg PL 508.

84 Der Inspekteur der Motorsportausbildung der HJ des Kraftfahr-Inspektionsbereiches Süd zerstreute in seinem Schreiben an die Motorstandarte 87 vom 12.4. 1935 die Bedenken des Vorsitzenden der Fahrlehrervereinigung von Schwaben und Neuburg; StA München, NSDAP 1310.

85 Schreiben der Korpsführung an den Reichsverkehrsminister vom 18.2. 1937, BArch NS 24/103.

86 Der NSKK-Mann vom 20. August: „Ritter vom Kleinmotor werden geschult“. 
wurden reichseinheitliche Strukturen geschaffen, auf die die Technischen Überwachungsvereine nach dem Zweiten Weltkrieg aufbauen konnten. ${ }^{87}$

Die Mitwirkung des NSKK an der Überwachung der Fahrlehrer und an der Neuzulassung der technischen Sachverständigen führte jedoch zu einer Gemengelage von Interessen und Machtstrukturen, die sich insgesamt lähmend auswirkte. Die Einführung weltanschaulicher Kriterien - politische Zuverlässigkeit und „arische" Herkunft - stellten einen Rückschritt in Bezug auf eine freizügige Berufsausübung dar.

Eine institutionelle Übernahme der Fahrlehrer und der technischen Sachverständigen in das Korps wäre einer Revolutionierung der staatlichen Verwaltung im Verkehrssektor gleichgekommen. Dieses Bestreben schlug fehl. Der Partei und ihren Gliederungen oblag, gemäß dem Gesetz zur Sicherung der Einheit von Partei und Staat vom 1. Dezember 1933, die „Menschenführung“, dem Staat jedoch die „Sachwaltung“. Sobald das NSKK in seinem Expansionsstreben die Grenzen als „Erziehungsschule der Motorisierung“ überschreiten wollte, rief es den Widerstand in Parteikreisen und von Seiten des Reichsverkehrsministeriums hervor. Im Neben- und Gegeneinander von NSKK und Reichsverkehrsministerium wird der Dualismus von Partei und Staat deutlich. Der Versuch des NSKK, die Autonomie der Fahrlehrerverbände und Technischen Überwachungsvereine zu durchbrechen, scheiterte am Widerstand der stärkeren Machtblöcke.

87 Technische und wirtschaftliche Gründe für die Neuordnung, also durchaus modernisierende Elemente, die auch nach dem Krieg ihre Geltung behielten, konstatierte auch Wiesenack in seiner Untersuchung: Wesen und Geschichte der Technischen ÜberwachungsVereine, S. 85. 